\title{
Assistência de enfermagem em diálise peritoneal: aplicabilidade da teoria de orem - estudo de método misto ${ }^{a}$
}

\author{
Asistencia em enfermería em diálisis peritoneal: aplicabilidad de la teoría de orem - \\ estudio de método mixto
}

Nursing assistance in peritoneal dialysis: applicability of orem theory -mixed method study

Denise Rocha Raimundo Leone ${ }^{1}$ (1)

Ana Carolina de Oliveira Jeronymo Neves $^{2}$ (D)

Roberta Teixeira Prado ${ }^{3}$ (1)

Edna Aparecida Barbosa de Castro ${ }^{4}$ (])

1. Universidade Federal de Juiz de Fora. Juiz de Fora, MG, Brasil.

2. Universidade Federal do Rio de Janeiro. Rio de Janeiro, RJ, Brasil.

3. Faculdade de Ciências Médicas e da Saúde de Juiz de Fora. Juiz de Fora, MG, Brasil.

4. Universidade Federal de Juiz de Fora, Programa de Pós-Graduação Stricto Sensu, Curso de Mestrado em Enfermagem. Juiz de Fora, MG, Brasil.
Autor correspondente:

Edna Aparecida Barbosa de Castro

E-mail: ednabdecastro@aol.com.

Recebido em 21/08/2020.

Aprovado em 30/10/2020.

DOl:https://doi.org/10.1590/2177-9465-EAN-2020-0334

\section{Resumo}

Objetivo: Compreender a aplicabilidade da Teoria Geral de Enfermagem de Orem na assistência prestada aos pacientes em diálise peritoneal domiciliar. Método: Estudo de método misto. Na etapa quantitativa realizou-se uma pesquisa transversal, descritiva e exploratória, com 34 pacientes em diálise peritoneal. Aplicou-se um instrumento para caracterização sociodemográfica e clínica e a escala de avaliação da capacidade para o autocuidado - Appraisal of Self Care Agency Scale Revised. Análise dos dados por estatística descritiva. Na abordagem qualitativa, utilizou-se a Teoria Fundamentada nos Dados. Realizaram-se 23 entrevistas domiciliares com 19 participantes. Análise dos dados seguiu as codificações aberta, axial e seletiva. Resultados: Dos participantes, $59 \%$ tinham capacidade para o autocuidado operacionalizado. Os pacientes em diálise peritoneal atenderam, em diferentes graus, às seis categorias de requisitos de autocuidado de desvio de saúde para a realização da terapia em domićilio. $\mathrm{O}$ apoio $e$ a educação destacaram-se como modalidade de sistema de Enfermagem. Conclusão e implicação para a prática: A Teoria Geral de Enfermagem de Orem se aplica na assistência de Enfermagem a pessoas em diálise peritoneal. Sugere-se sua utilização como suporte teórico para o Processo de Enfermagem.

Palavras-chave: Autocuidado; Diálise Peritoneal; Enfermagem; Teoria de Enfermagem; Nefrologia

\section{Abstract}

Objective: To understand the applicability of Orem's General Nursing Theory in assisting patients on peritoneal dialysis at home. Method: Mixed method study. In the quantitative stage, a cross-sectional, descriptive and exploratory study was carried out with 34 patients on peritoneal dialysis. An instrument was applied for sociodemographic and clinical characterization and the scale for assessing the capacity for self-care - Appraisal of Self Care Agency Scale Revised. Analysis of data using descriptive statistics. In the qualitative approach, Grounded Theory was used. 23 home interviews were conducted with 19 participants. Data analysis followed open, axial and selective coding. Results: $59 \%$ of the participants had the capacity for operationalized self-care. Patients on peritoneal dialysis met, to varying degrees, the six categories of self-care requirements for health deviation to perform therapy at home. Support and education stood out as a modality of the Nursing system. Conclusion and implication for practice: Orem's General Nursing Theory applies to nursing care for people on peritoneal dialysis. Its use is suggested as a theoretical support for the Nursing Process.

Keywords: Self-care; Peritoneal Dialysis; Nursing; Nursing Theory; Nephrology.

\section{Resumen}

Objetivo: comprender la aplicabilidad de la teoría general de enfermería de Orem para ayudar a los pacientes en diálisis peritoneal en el hogar. Método: estudio de método mixto. En la etapa cuantitativa, se realizó un estudio transversal, descriptivo y exploratorio con 34 pacientes en diálisis peritoneal. Se aplicó un instrumento para la caracterización sociodemográfica y clínica y la escala para evaluar la capacidad de autocuidado - Evaluación de la escala de la agencia de autocuidado revisada. Análisis de datos mediante estadística descriptiva. En el enfoque cualitativo, se utilizó la teoría fundamentada. Se realizaron 23 entrevistas domiciliarias con 19 participantes. El análisis de datos siguió una codificación abierta, axial y selectiva. Resultados: de los participantes $59 \%$ tenían la capacidad de autocuidado operacionalizado. Los pacientes en diálisis peritoneal cumplieron, en diversos grados, las seis categorías de requisitos de autocuidado para la desviación de salud para realizar la terapia en el hogar. El apoyo y la educación se destacaron como una modalidad del sistema de enfermería. Conclusión e implicación para la práctica: la teoría general de enfermería de Orem se aplica a los cuidados de enfermería para personas en diálisis peritoneal. Su uso se sugiere como soporte teórico para el Proceso de Enfermería.

Palabras clave: Autocuidado; Diálisis Peritoneal; Enfermería; Teoría de Enfermería; Nefrología. 


\section{INTRODUÇÃO}

A diálise peritoneal (DP) é um tipo de terapia renal substitutiva realizada como tratamento para doença renal crônica (DRC) com necessidade dialítica. ${ }^{1}$ No Brasil, estima-se que há 10.410 pessoas em tratamento por $\mathrm{DP},{ }^{2}$ sendo a prevalência de pessoas que realizam esta terapia de 45,6 por milhão de população (pmp). Esta é menor quando comparada com países como El Salvador (288,7 pmp), Guatemala (221,3 pmp), Colômbia (143,6 pmp), Uruguai (71,6 pmp) e Chile (61,2 pmp) e maior em relação à Argentina (36 pmp) e Paraguai (4pmp). ${ }^{3}$

ADP possui duas modalidades, a diálise peritoneal ambulatorial contínua (CAPD), que é um método manual, no qual é realizada a troca da solução de diálise da cavidade peritoneal por meio da gravidade, a cada 4-5 horas, e a diálise peritoneal automatizada (DPA), que é efetivada por meio de uma máquina responsável pelo processo de troca da solução de diálise por 8 a 10 horas por noite, deixando o paciente livre durante o dia para outras atividades. ${ }^{4}$

Independentemente do método utilizado, a DP pode ser realizada em domicílio pelo próprio paciente ou com a ajuda de um cuidador. ${ }^{4}$ Para tanto, os mesmos devem participar da educação terapêutica para o autocuidado, que inclui: o procedimento da diálise, a atuação e reconhecimento de intercorrências, o ganho de peso entre as sessões de DP e a dieta adequada. ${ }^{5}$ Sendo assim, ela precisa ter a capacidade para o autocuidado operacionalizada, ou seja, ter a capacidade para realizar seu autocuidado. ${ }^{6}$

Além disso, trata-se de um tratamento domiciliar, em que o paciente em DP deve comparecer periodicamente às consultas de Enfermagem para acompanhamento. ${ }^{5}$ Tanto nas consultas de Enfermagem quanto na educação terapêutica, o enfermeiro se insere como facilitador dos cuidados do paciente em DP. Pois, além de executar procedimentos de Enfermagem, ele orienta e avalia os aspectos cognitivos, sociais, emocionais, ambientais e àqueles relacionados ao tratamento, como dieta, utilização correta da medicação e destreza manual. ${ }^{7}$ Neste sentido, a assistência ofertada deve ser sistematizada por meio do Processo de Enfermagem ancorado em um suporte teórico, ${ }^{8}$ a fim de evitar lacunas e divergências conceituais impeditivas da eficiência e qualidade assistencial. ${ }^{9}$

Neste contexto, a Teoria de Enfermagem de Orem tem sido utilizada na assistência de Enfermagem a pacientes com doenças crônicas. ${ }^{9,10}$ Ressalte-se que esta é um constructo teórico que abarca três teorias, a Teoria do Autocuidado, a Teoria do Déficit do Autocuidado e a Teoria dos Sistemas de Enfermagem, ${ }^{6}$ e a sua aplicabilidade pode ser expandida a diversos indivíduos e grupos com necessidades de autocuidado. ${ }^{9}$

A teoria do autocuidado engloba conceitos necessários ao seu entendimento, são eles: autocuidado, ações de autocuidado, exigências terapêuticas e requisitos para o autocuidado. ${ }^{6,11}$

Define-se por autocuidado "a prática de atividades que os indivíduos iniciam e executam em seu favor, na manutenção da vida, saúde e bem-estar". $6: 104$ Já as ações de autocuidado, também denominadas agência do autocuidado, referem-se ao poder e à capacidade que o indivíduo possui para se engajar no autocuidado. ${ }^{6,12}$
As ações realizadas pelas pessoas com o objetivo de cuidar de si são denominadas exigências terapêuticas e devem ser efetivadas por um período específico de tempo, a fim de satisfazer os requisitos do autocuidado. Dentre os requisitos, têm-se: os requisitos universais, que englobam os processos necessários para a integridade do corpo, como respirar e se alimentar; os requisitos desenvolvimentais que são voltados para o desenvolvimento da pessoa e que se modificam conforme os diferentes estágios da vida; e os requisitos de desvio de saúde que se referem aos cuidados ou tomadas de decisões que são exigidas em casos de doença, com o objetivo de recuperação, reabilitação e controle. Vale ressaltar que as intervenções de Enfermagem, geralmente, possuem como foco os requisitos de desvio de saúde e que estes exigem o autocuidado terapêutico. ${ }^{6}$

São seis os requisitos de autocuidado de desvio de saúde:6:201-202

1) Buscar e garantir assistência médica apropriada em casos que possam comprometer a saúde como a exposição a agentes biológicos ou a condições físicas, ambientais, genéticas e/ ou psicológicas que possam estar associadas a patologias humanas.

2) Estar consciente em relação aos efeitos e resultados da condição patológica.

3) Realizar efetivamente as medidas diagnósticas, terapêuticas e de reabilitação prescritas, direcionadas à prevenção, recuperação e controle de determinados tipos de patologia.

4) Ter conhecimento, observar e regular os efeitos colaterais e desconfortáveis provenientes das medidas de tratamento médico, incluindo os efeitos sobre o desenvolvimento.

5) Aceitar e adaptar-se positivamente às suas adversidades de saúde e, consequentemente, aderir às formas específicas de atendimento.

6) Aprender a viver com os efeitos das suas condições patológicas, promovendo o desenvolvimento pessoal de forma sistemática e contínua.

Destaca-se que a demanda de autocuidado terapêutico se refere ao tipo e quantidade de cuidados que são prescritos, por um período de tempo, visando a manutenção da vida. ${ }^{6}$ Sendo que, na Teoria do Autocuidado, quando a necessidade do autocuidado terapêutico é superior à capacidade da realização das ações de autocuidado do indivíduo, tem-se, então, o déficit de autocuidado, no qual a assistência da Enfermagem é exigida. ${ }^{11}$

Nesta pesquisa, para verificar a aplicabilidade da Teoria de Enfermagem de Orem, o foco foi a demanda de autocuidado terapêutico e os requisitos de autocuidado de desvio de saúde, visto que os pacientes que faziam DP domiciliar realizavam atividades prescritas por profissionais de saúde para a manutenção da vida.

A Teoria do Déficit do Autocuidado versa sobre os casos em que o indivíduo não consegue suprir todas as demandas necessárias para satisfazer seu autocuidado ou quando se encontra diante de limitações, evidenciando a necessidade da Enfermagem. Assim, ela deve utilizar um dos cinco métodos de ajuda, a saber: agir ou fazer para o outro; guiar ou orientar; apoiar física e psicologicamente; propiciar e manter um ambiente de apoio ao desenvolvimento pessoal ou ensinar o outro..$^{6,12}$ 
A Teoria dos Sistemas de Enfermagem, por sua vez, descreve as possibilidades de assistência às pessoas com déficit de autocuidado, conforme a classificação dos sistemas de Enfermagem, sendo eles: o sistema totalmente compensatório: quando a Enfermagem é responsável por satisfazer todas as demandas que garantirão a existência humana; sistema parcialmente compensatório: nos casos em que tanto a Enfermagem quanto a pessoa executam atividades de autocuidado; sistema de apoio-educação, que ocorre quando a pessoa é capaz de realizar as atividades de autocuidado, porém necessita aprender as atividades de autocuidado terapêutico que são ensinadas pelos enfermeiros. . $^{6,12}$

Neste contexto, quando analisada a aplicação deste constructo como arcabouço teórico do Processo de Enfermagem em nefrologia, percebe-se a utilização de parte deste, com uso de uma ou duas teorias ou de conceitos imbuídos nestas, como o conceito do autocuidado, ${ }^{13-15}$ ou referindo-se aos pacientes em hemodiálise. ${ }^{16}$

Com isso, é relevante que se explicite a sua aplicabilidade nesta área, possibilitando a Sistematização da Assistência de Enfermagem, a construção de projetos coletivos e discursos próprios da disciplina de Enfermagem. ${ }^{9}$ Justificou-se, portanto, este estudo pelo potencial do constructo teórico de Orem em dar visibilidade e fundamentar o processo de cuidar, relacionado com a doença e o tratamento, que vise à promoção do autocuidado e à autonomia do paciente renal crônico e família.

Sendo assim, levantou-se a seguinte questão norteadora: como a Teoria de Enfermagem de Orem se aplica à assistência de enfermagem prestada a pacientes em DP? Objetivou-se, portanto, compreender a aplicabilidade da Teoria Geral de Enfermagem de Orem à assistência prestada aos pacientes em DP domiciliar.

\section{MÉTODO}

Os resultados divulgados neste artigo foram extraídos da dissertação de mestrado em Enfermagem intitulada: "Diálise Peritoneal Domiciliar: habilidades para a realização do ritual terapêutico".

Este artigo apresenta um estudo com método misto pela estratégia transformador sequencial, em que a pesquisa ocorre sequencialmente em duas fases, adotando uma lente teórica para guiar o estudo e sobrepondo-se à sequência desde a coleta dos dados. ${ }^{17}$

Neste estudo, a primeira fase foi com abordagem quantitativa e a segunda com a qualitativa, com prioridade da perspectiva qualitativa (quan $\rightarrow$ QUAL), guiadas pela Teoria de Doroteia Orem como perspectiva teórica para a integração na análise/discussão dos resultados. Desta forma, na primeira fase realizou-se uma pesquisa transversal, descritiva e exploratória visando avaliar a capacidade do autocuidado dos participantes diante da demanda por DP domiciliar. Aplicou-se um questionário estruturado, de elaboração própria, para caracterização sociodemográfica e clínica e uma escala para avaliação da capacidade do autocuidado, a Appraisal of Self Care Agency Scale Revised (ASAS-R). A ASAS-R foi validada para utilização no Brasil e é uma escala do tipo Likert, que contém 15 questões com 5 possibilidades de resposta, que varia de discordo totalmente a concordo totalmente. $O$ intervalo de pontuação vai de 01 a 75, sendo que quanto maior a pontuação maior a operacionalização para a capacidade do autocuidado. ${ }^{18,19}$ $\mathrm{Na}$ fase quantitativa, a análise dos dados se deu por meio de estatística descritiva, sendo calculada a média, desvio-padrão, frequência relativa e absoluta. Utilizou-se o software Statistical Package for the Social Science (SPSS) versão 20.0. Os dados desta fase possibilitaram, também, selecionar e caracterizar grupos amostrais para a etapa qualitativa.

$\mathrm{Na}$ segunda fase, a abordagem qualitativa seguiu as diretrizes da Teoria Fundamentada nos Dados (TFD) Straussiana, um método de tradição pós-positivista que visa, ao invés de testar, desenvolver teoria derivada de dados sistematicamente analisados, em constantes comparações entre si. Caracteriza-se por utilizar amostragem teórica, análise comparativa constante dos dados, elaboração de memorandos e diferenciar teoria substantiva de teoria formal. ${ }^{20} \mathrm{~A}$ vertente Starussiana possibilita o uso de referencial teórico; a coleta, análise dos dados e teoria são intrinsecamente imbricados, extraindo-se um esquema inovador que reflete melhor a realidade estudada. ${ }^{21} \mathrm{O}$ objetivo desta etapa foi compreender o atendimento aos requisitos de desvio de autocuidado pelos pacientes em DP.

Considerando-se as duas fases, a coleta de dados ocorreu entre junho de 2015 e junho de 2016 e em dois cenários. O primeiro foi o serviço de diálise de um hospital público de ensino do Estado de Minas Gerais, com capacidade de atendimento para até 50 pacientes em DP, onde os participantes do estudo estavam cadastrados para a realização do tratamento. $O$ segundo cenário foi o domicílio dos participantes da pesquisa.

Antes da coleta de dados, a pesquisadora realizou visitas semanais no serviço de diálise pelo período de cinco meses, por entender que nestes momentos seriam criadas e aprimoradas as relações interpessoais que favoreceriam a posterior coleta de dados empíricas no domicílio. Naqueles momentos, os pacientes cadastrados no serviço de DP foram informados que a pesquisadora era enfermeira e que faria uma pesquisa, ficando os mesmos cientes dos objetivos da pesquisa, além de realizarem a leitura e assinatura do TCLE.

No serviço de DP, das 37 pessoas cadastradas, três foram excluídas: uma por déficit cognitivo relatado pela cuidadora $e$ confirmado pela pesquisadora, o que a impedia de responder a entrevista, e duas pessoas por óbito. Sendo assim, participaram da primeira fase 34 pacientes.

Adotaram-se como critérios de inclusão: pessoas maiores de 18 anos, que fossem capazes de se expressar por meio de linguagem verbal; que realizassem DP no domicílio e estivessem cadastradas no serviço de DP, cenário desta investigação. Foram excluídos àqueles que não tinham condições para responder a pesquisa, de acordo com a avaliação realizada pelo entrevistador no ato da pesquisa e ratificado pelo cuidador que o acompanhava; e para a segunda etapa da pesquisa, àqueles que residiam em outro município.

Na segunda fase, dentre os possíveis participantes, 14 foram excluídos, pois oito residiam em outro município, três foram a óbito, dois foram transferidos para hemodiálise e um não aceitou 
receber a visita domiciliar (VD) para a realização da entrevista. Nesta, os dados foram coletados em domicílio utilizando as técnicas de entrevista aberta, observação participante, registros do diário de campo e elaboração de memorandos. As entrevistas tiveram duração aproximada de 30 minutos, foram gravadas e posteriormente transcritas.

Dezenove pessoas participaram da segunda fase. As mesmas foram distribuídas em três grupos amostrais. No primeiro participaram dez pessoas e as entrevistas foram guiadas pelas perguntas: "Para você, como é fazer diálise peritoneal em casa?"; "Fale para mim quais são os passos que você faz para realizar uma sessão de diálise?"; "Para você, se existirem, quais são as facilidades em realizar a diálise peritoneal?"; "Existem dificuldades? Quais?"; "Quais os cuidados que você tem para melhorar seu tratamento?"; "Recebe ou já recebeu visita da Equipe da Saúde da Família ou Equipe Multiprofissional da Atenção Domiciliar? Se sim, como foi?".

Com a análise dos dados e com o objetivo da obtenção da saturação teórica, surgiu a necessidade de um segundo grupo amostral, para o qual utilizaram-se as seguintes questões norteadoras: "Como é o seu dia a dia? Conta para mim, o que você faz da hora em que você acorda até a hora em que você dorme"; "Como você se organiza e encaixa a diálise no seu dia a dia?"; "O que ajuda você a conviver com a DP e a realizá-la?". Este foi composto de quatro participantes.

Assim, obteve-se a saturação teórica no decurso da coleta de dados pelas visitas domiciliares e entrevistas a 14 participantes. Com a necessidade da validação teórica, surgiu o terceiro grupo amostral, composto de nove pessoas, sendo que destas, quatro também compuseram o primeiro grupo, totalizando-se 23 entrevistas com 19 participantes. Salienta-se que o tamanho amostral se orientou pelo critério de saturação teórica, ou seja, coletaram-se os dados até que as categorias estivessem bem desenvolvidas no que se refere às suas propriedades e dimensões. ${ }^{21}$

A análise foi realizada concomitantemente à coleta e ocorreu segundo três tipos de codificação: aberta, axial e seletiva. A primeira consistiu na identificação dos conceitos expressos nos dados e foi realizada por meio da técnica de microanálise das transcrições das entrevistas. A codificação axial buscou estabelecer relação entre as categorias e suas subcategorias e, por meio da codificação seletiva, integrou-se e refinou-se uma teoria substantiva sobre o fenômeno pesquisado, apresentada na forma de um diagrama, no qual se buscou identificar a relação entre a categoria central e as subcategorias. ${ }^{21}$

Após a análise separada dos dados quantitativos e qualitativos, realizou-se a integração dos mesmos pela estratégia transformadora sequencial. ${ }^{77}$ Foram identificadas as similaridades e diferenças nos dados da etapa qualitativa, conforme a classificação do paciente quanto à sua capacidade para operacionalização do autocuidado, ou seja, realizou-se uma análise complementar. Esta objetivou comparar o atendimento dos requisitos de autocuidado de desvio de saúde dos pacientes com capacidade para o autocuidado operacionalizado com àqueles classificados como capacidade para o autocuidado não operacionalizado. Possibilitando a compreensão de como a Teoria de Enfermagem de Orem se aplica à assistência de enfermagem prestada aos pacientes em DP. Para a organização do banco de dados empíricos, edição textual e apoio à codificação, utilizou-se o software OpenLogos ${ }^{\circledR}$.

Foram atendidos todos os requisitos éticos, segundo a Resolução n. 466/2012 do Conselho Nacional de Saúde. ${ }^{22}$ A pesquisa foi aprovada por um Comitê de Ética sob o parecer $\mathrm{n}^{\circ}$. 1.004.325 e, para preservar o anonimato, os participantes foram cognominados por meio de nomes de flores.

\section{RESULTADOS}

\section{Caracterização dos participantes}

As características sociodemográficas e clínicas estão listadas na Tabela 1. Destaca-se que $79,5 \%$ são mulheres, $53 \%$ são analfabetos ou possuem o ensino fundamental incompleto, $94,1 \%$ são beneficiários do Instituto Nacional de Seguro Social (INSS). A idade variou entre 32 e 89 anos, sendo a média $56,62(+14,62)$ anos. Os demais dados sociodemográficos e clínicos se encontram na Tabela 1.

Quanto à operacionalização do autocuidado, a pontuação da escala ASAS-R variou entre 44 e 64, sendo a média de54 $(+6,47)$ pontos. Deste modo, 59\% (20) dos participantes tinham capacidade para o autocuidado operacionalizado e 41\% (14) não tinham capacidade para o autocuidado operacionalizado. Salienta-se que entre os sete participantes da pesquisa do sexo masculino, quatro (57\%) tinham capacidade para o autocuidado não operacionalizado. Já entre as 27 mulheres, 37\% $(n=10)$ não tinham operacionalização para o autocuidado.

\section{Aplicabilidade da Teoria Geral de Enfermagem de Orem na assistência a pessoas em DP: atendimento aos requisitos de desvio de saúde e classificação quanto ao sistema de Enfermagem}

Para que a pessoa possa realizar DP domiciliar, torna-se necessária a realização de atividades de autocuidado terapêutico e, para tanto, a mesma precisa ter a capacidade de se engajar no autocuidado. Salienta-se que, mesmo contando-se com um ajudante ou responsável pelo procedimento dialítico, o convívio com a doença e suas exigências requer que o paciente mantenha outras atividades de autocuidado terapêutico como, por exemplo, realização de curativo diário no óstio do cateter, conforme relatado por Dália.

[...] Eu faço curativo todo dia. Desde quando vim para casa, eu nunca abandonei a tarefa de fazer o curativo. Na hora do banho, eu lavo bem lavado, passo álcool 70\% e pomada, eu coloco. A doutora me dá e eu coloco [...] (Dália).

Ao considerar a capacidade para a realização do autocuidado com as demandas de autocuidado terapêutico, percebeu-se que os participantes classificados como tendo a capacidade para o autocuidado não operacionalizado necessitaram de auxílio de um familiar ou relataram maior preocupação em realizar a 
Tabela 1. Caracterização sociodemográfica e clínica de pessoas em diálise peritoneal, Juiz de Fora, Minas Gerais, Brasil.

\begin{tabular}{|c|c|c|c|c|}
\hline \multirow{2}{*}{$\begin{array}{ll} & \text { Dados sociodemográficos } \\
\text { Sexo Feminino } & \end{array}$} & \multicolumn{2}{|c|}{$\% \mathrm{~N}$} & \multirow{2}{*}{$\begin{array}{c}\text { Média } \\
-\end{array}$} & \multirow{2}{*}{$\begin{array}{c}\text { DP } \\
-\end{array}$} \\
\hline & $79,5 \%$ & 27 & & \\
\hline Sexo Masculino & $20,5 \%$ & 7 & - & - \\
\hline \multicolumn{5}{|l|}{ Escolaridade } \\
\hline Analfabeto/ensino fundamental incompleto & $53 \%$ & 18 & - & - \\
\hline Ensino fundamental completo & $5,8 \%$ & 2 & - & - \\
\hline Ensino médio completo & $35,3 \%$ & 12 & - & - \\
\hline Ensino superior completo & $5,9 \%$ & 2 & - & - \\
\hline \multicolumn{5}{|l|}{ Ocupação } \\
\hline Atividade laborativa & $5,9 \%$ & 2 & - & - \\
\hline Beneficiários do Instituto Nacional de Segurança Social & $94,1 \%$ & 32 & - & - \\
\hline \multicolumn{5}{|l|}{ Renda Pessoal } \\
\hline Menor que 1 salário mínimo & $2,9 \%$ & 1 & - & - \\
\hline 1 a 2 salários mínimos & $85,4 \%$ & 29 & - & - \\
\hline 3 a 4 salários mínimos & $8,8 \%$ & 3 & - & - \\
\hline \multicolumn{5}{|l|}{ Dados Clínicos } \\
\hline \multicolumn{5}{|l|}{ Tipo de Diálise } \\
\hline Diálise Peritoneal Automatizada & $94,1 \%$ & 32 & - & - \\
\hline Diálise Peritoneal Ambulatorial Contínua & $5,9 \%$ & 2 & - & - \\
\hline Tempo em meses & - & - & 25,5 & +18 \\
\hline \multicolumn{5}{|l|}{ Peritonite no último ano } \\
\hline Não & $94,1 \%$ & 32 & - & - \\
\hline Sim & $5,9 \%$ & 2 & - & - \\
\hline Necessitam de ajuda para realizar a diálise peritoneal & 64,7 & 22 & - & - \\
\hline
\end{tabular}

Fonte: Dados de pesquisa.

diálise no domicílio, além de apresentarem maior dificuldade em relatar os passos necessários à instalação da diálise, quando comparados com àqueles considerados com capacidade para o autocuidado operacionalizado.

No que tange aos requisitos de desvio de saúde, apreendeu-se que todos os participantes se engajam nas exigências terapêuticas de autocuidado e atendem, em diferentes graus, às seis categorias de requisitos de saúde. Para satisfazer o primeiro requisito, o indivíduo precisa buscar e assegurar assistência médica apropriada, quando necessário. Nesta, percebeu-se que os pacientes em DP, por realizarem o tratamento em domicílio, estão conscientes de suas necessidades de saúde e buscam a ajuda especializada, conforme relatado por Amarílis e Orquídea e, desta forma, cumprem as exigências deste requisito.

[...]Se der problema na máquina, eu desligo a máquina e vou dormir, aí, no dia seguinte, eu ligo ou venho aqui [serviço de nefrologia], porque aqui não funciona à noite [...] (Amarílis).
[...] Umas duas vezes, à noite, eu já liguei para a XXX [empresa que fornece a cicladora e materiais para a realização da DP que tem um telefone de serviço de atendimento ao cliente], mas não adiantou nada, não conseguiram resolver o problema, então eu desliguei a máquina e, no dia seguinte, trouxe ela aqui [serviço de nefrologia] [...] [(Orquídea).

O segundo requisito de desvio de saúde, diz respeito à consciência relacionada aos efeitos e resultados da doença. E, com a análise dos dados, apreendeu-se que o conviver com a necessidade de realizar DP faz com que os pacientes vivenciem incômodos e temores relacionados ao tratamento, conforme relato de Centáurea.

[...] No início, a gente ficava muito preocupada, eu tinha medo, não tinha certeza se estava fazendo certo, se o tratamento ia dar certo, porque era um tratamento novo. Hoje não, é tudo tranquilo [...] (Centáurea). 
Além disso, os participantes percebiam que a DRC os limitava fisicamente. Apreendeu-se que as limitações variavam conforme o paciente e que os mesmos realizavam adaptações, de acordo com a fala de Crisântemo.

[...] Mesmo fazendo o tratamento, eu não deixo de fazer certas atividades, porque eu acho que isso espairece e tal. É lógico que eu não vou ficar pegando peso, sabendo que eu não posso ficar pegando peso, mas eu faço algumas atividades [...] (Crisântemo).

Além das adaptações relatadas, os participantes afirmaram desenvolver atividades de autocuidado terapêutico que englobam, entre outras, o cuidado com o cateter de DP, o que é exemplificado na fala de Centáuria, o tratamento medicamentoso e não medicamentoso, no qual se destaca a realização de dieta adequada, o manejo da ingestão de líquidos e a realização de atividade física, conforme relatos de Tulipa e Azaleia.

[...] Eu tomo banho e lavo bem lavadinho, com sabonete, 0 orifício. Eu às vezes jogo álcool [...] (Centáurea).

[...] Eu até vou à Chácara fazer ginástica, mas uma vez por semana [...] (Tulipa).

[...] Ah, eu evito comer muito, mas dieta eu não faço não. Eu evito comer muito, eu como de tudo, mas evito comer muito [...] (Azaleia).

O procedimento da DP também foi incluído nas atividades de autocuidado terapêutico. Percebeu-se que se trata de um processo sistematizado, conforme a fala de Lírio, a qual envolve muito além da conexão do paciente com o sistema da DP, mas todo um preparo do ambiente, dos recursos materiais e das pessoas envolvidas até se findar na diálise em si.

[...] Primeiro, eu separo todo o material, não é, depois eu limpo a máquina com pano úmido e depois limpo a mesa com álcool. Depois limpo todo material, as bolsas, o cassete, o acesso de drenagem, todas as pecinhas com álcool e uma toalhinha. E depois eu lavo a mão de novo e vou conectar à máquina, depois conecto as bolsas, né? Ponho para preencher as linhas, venho, e faço a última higiene e deito para esperar a linha preencher para poder me ligar [...] (Lírio).

O desenvolvimento destas atividades de autocuidado terapêutico faz com que os pacientes cumpram o terceiro requisito de desvio de saúde, pois estas tratam da realização das medidas terapêuticas da DRC.

O quarto requisito diz respeito a aprender a viver com as adversidades, além de promover o desenvolvimento pessoal. $\mathrm{E}$, com a análise dos dados ratifica-se a peculiaridade do atendimento que deve ser fornecido a este, pois os participantes em DP têm consciência de sua situação de saúde e procuram desenvolver alguma atividade e/ou manter alguma ocupação, visando atenuar o convívio com a doença, além de promover o desenvolvimento pessoal, conforme relato de Crisântemo.

[...] É lógico que eu não vou ficar pegando peso, sabendo que eu não posso ficar pegando peso, mas eu faço algumas atividades. Eu gosto de cozinhar, eu vou para a cozinha fazer as coisas. E trabalhos extras e tal que precisam ser feitos, eu faço [...] (Crisântemo).

Percebe-se com a fala de Crisântemo que, além de ter aprendido a conviver com as adversidades, o mesmo se adaptou às atividades que desenvolve, pois não realiza atividades que envolvam "pegar peso" e desta forma, cumpre-se também o quinto requisito de desvio de saúde.

No que tange às adaptações, foi possível perceber que todos os participantes necessitaram adaptar suas residências, seja com a construção de banheiros e retirada de cortinas, seja com objetos de decoração, conforme as falas de Calas e Dália.

[...] Colocou parede no quarto, colocou uma porta, a cortina teve que mudar [...] (Calas).

[...] Eu fiz um quadradinho, um banheiro, um vaso com uma pia, entendeu? [...] (Dália).

O empenho dos pacientes em realizarem as adaptações tanto no seu cotidiano quanto em seus domicílios demonstra que os mesmos atenderam ao quinto e sexto requisito do desvio de saúde, que são, respectivamente, aceitar e se adaptar positivamente às suas adversidades de saúde, ter conhecimento regular e observar os efeitos colaterais do tratamento.

Diante do exposto, reitera-se que pessoas em DP domiciliar satisfazem, em diferentes graus, todos os requisitos de autocuidado terapêutico definidos por Orem e, para tanto, os mesmos se qualificaram através da educação terapêutica fornecida pelo enfermeiro do serviço de DP. Neste sentido, compreende-se que o Sistema de Enfermagem presente neste contexto é o de apoio-educação.

Salienta-se que, caso o paciente não apresente capacidade para o autocuidado operacionalizado e que não possua um responsável para a realização do procedimento dialítico, o mesmo deverá ser submetido ao tratamento no serviço de DP sob os cuidados do enfermeiro responsável. Nestes casos, o sistema de Enfermagem presente será o Sistema Pacialmente Compensatório.

\section{DISCUSSÃO}

O perfil sociodemográfico e clínico da amostra foi semelhante àqueles descritos em outras pesquisas nacionais ${ }^{23,24} \mathrm{e}$ internacionais. Entretanto, na literatura internacional predominaram os pacientes em CAPD em detrimento da DPA. ${ }^{25,26}$

Quanto à operacionalização do autocuidado, nesta amostra, a média de pontos na escala ASAS-R foi de $54(+6,47)$, semelhante 
à que foi encontrada em pesquisa brasileira, que adaptou e validou a escala para o contexto brasileiro e realizada com 240 pacientes diabéticos $(56,8+0,48)^{19}$ e em investigação espanhola com 106 pacientes com lesão da medula espinhal $(50,87+6,83) \cdot{ }^{27}$ Entretanto, este foi superior ao resultado em amostra com 309 pacientes hipertensos na Etiópia $(27,8+7,0) \cdot{ }^{28}$ Este achado expõe a existência de relação entre as repercussões da doença de base e o conexto local do paciente na operacionalização do autocuidado.

Já em pesquisa realizada no Brasil, com 100 pacientes em tratamento hemodialítico, a média de pontos na escala foi de $60,64(+8,24) .{ }^{29}$ Até onde sabemos, não há estudos nacionais e internacionais sobre capacidade do autocuidado em pacientes em DP, sendo necessárias novas pesquisas para ampliar essa compreensão.

Nesta investigação, 59\% dos participantes apresentaram capacidade para o autocuidado operacionalizado. Há evidências de que entre pacientes hipertensos há, também, o predomínio $(52,8 \%)$ de pessoas com boa agência do autocuidado. ${ }^{28}$ Apreende-se que há importante espaço para a atuação da enfermagem junto às pessoas com capacidade do autocuidado não operacionalizado, visando melhorá-la por meio do processo de cuidar sistematizado.

Salienta-se que a utilização da escala ASAS-R possa ser uma estratégia a ser utilizada por enfermeiros, a fim de direcionar a assistência prestada e prescrita, uma vez que uma boa capacidade para o autocuidado aumenta a probabilidade de o paciente possuir boas práticas de autocuidado. ${ }^{28}$ Neste contexto, ao utilizar a Teoria de Enfermagem de Orem como fundamento teórico no Processo de Enfermagem, no atendimento aos pacientes em DP, torna-se interessante a aplicação da escala ASAS-R.

No que se refere aos requisitos de autocuidado em desvio de saúde, apreendeu-se que os pacientes em DP satisfazem, em diferentes graus, às seis categorias dos requisitos. E, ao analisar a capacidade para o autocuidado investigada na primeira fase da pesquisa e o atendimento às demandas de autocuidado terapêutico, resultantes da etapa qualitativa, encontrou-se, ao integrar os resultados, que os pacientes com capacidade para o autocuidado não operacionalizado, quando comparados àqueles com capacidade para o autocuidado operacionalizado, dependiam do auxílio de um familiar ou cuidador para a realização do procedimento dialítico. Neste sentido, entende-se que estes não atenderam eficazmente o terceiro requisito de desvio de saúde, que diz respeito à realização das medidas diagnósticas e terapêuticas, requerendo mais atenção e a inclusão das ações de apoio e educação no plano de cuidados do enfermeiro.

Esse achado expressou a integração das análises das duas fases desta pesquisa, possibilitando compreender as diferenças e similaridades no atendimento aos requisitos de desvio de saúde pelos pacientes em DP.

O atendimento aos requisitos de desvio de saúde também foi investigado em pesquisa envolvendo pessoas com linfodemas de mama e nesta foram identificados três requisitos de autocuidado de desvio de saúde, foram eles os requisitos dois, três e seis. ${ }^{30}$
Embora não tenham sido encontrados outros estudos que dizem respeito ao atendimento dos requisitos de desvio de saúde por pacientes em DP, há relatos em outras amostras que os pacientes em DP procuram ajuda profissional quando necessário, pois se deslocam, o mais precocemente possível, para o serviço de diálise,${ }^{31}$ o que permite inferir que estes também atendem o primeiro requisito de desvio de saúde.

O receio pela necessidade do convívio com a diálise e a consciência da necessidade do tratamento, que acarreta mudanças e adaptações impostas ao indivíduo e sua doença, foram relatadas neste e em outros estudos, ${ }^{32,33}$ confirmando que os pacientes estavam conscientes da sua doença e dos resultados provenientes do adoecimento, demonstrando também que os mesmos aprenderam a viver com a doença para promover o desenvolvimento pessoal, atendendo, assim, ao segundo e ao quarto requisito de desvio de saúde.

Gerenciar o tratamento da DRC vai além da realização do tratamento dialítico em si, uma vez que existem outras demandas de autocuidado terapêutico, que incluem o cuidado com o cateter, tratamento medicamentoso e não medicamentoso, no qual se destacam restrição de líquidos, a manutenção de dieta adequada e a realização de atividade física. O cumprimento destas, seja parcialmente, seja totalmente foram relatadas em outros estudos, ${ }^{14,31}$ o que nos permite inferir o atendimento, em outras amostras, do terceiro requisito do desvio de saúde.

A necessidade de adaptação da residência para a realização da DP no domicílio e da adequação das exigências do tratamento à rotina dos pacientes em DP e de seus familiares foram descritas no estudo em tela e em outras pesquisas. ${ }^{31,34-36}$

Neste contexto, percebe-se um esforço dos pacientes em DP, com a ajuda de seus familiares, em aprenderem a conviver e se adaptarem positivamente às suas adversidades de saúde e demonstram que os mesmos possuem conhecimento sobre a doença e regulam os efeitos colaterais do tratamento e, dessa forma, atendem o quarto, quinto e sexto requisito de desvio de saúde. Vale salientar que a adaptação visa superar as dificuldades vivenciadas no cotidiano de pessoas em DP e estas devem ser avaliadas e estimuladas pelo enfermeiro na educação terapêutica para a DP. ${ }^{37}$

Destaca-se que a educação terapêutica, sendo de reponsabilidade do enfermeiro, é um recurso imperativo para o atendimento dos requisitos de desvio de saúde, pois, visa tornar o paciente independente e capaz de realizar o tratamento em domicílio. ${ }^{38,39}$ Além disso, como é o paciente quem realiza o procedimento dialítico no domicílio, identifica-se o sistema de Enfermagem de apoio-educação.

Diante do exposto, apreende-se a aplicabilidade da Teoria Geral de Enfermagem de Orem na assistência de Enfermagem de pacientes em DP, uma vez que este constructo instrumentaliza os profissionais para a assistência de qualidade, considerando o binômio saúde-doença. ${ }^{40}$ Além disso, mostrou-se adequado para utilização no Processo de Enfermagem, viabilizando a Sistematização da Assistência de Enfermagem às pessoas 
em DP, contribuindo para a prática de enfermagem baseada em evidência.

\section{CONCLUSÕES E IMPLICAÇÕES PARA A PRÁTICA}

Apreendeu-se que os pacientes em DP empenham esforços para garantir a realização das atividades de autocuidado terapêutico prescritas pelos profissionais de saúde. Desta forma, eles demostram satisfazer, em diferentes graus, às seis categorias de requisitos de autocuidado de desvio de saúde. Sendo que àqueles que possuem capacidade para o autocuidado não operacionalizado atendem em menor grau estes requisitos, quando comparado aos pacientes com autocuidado operacionalizado. Também, no que tange à Teoria dos Sistemas de Enfermagem, considerando todo o contexto da diálise domiciliar, está presente o sistema de apoio-educação.

A Teoria de Enfermagem de Orem engloba toda a complexidade que envolve a assistência do paciente em DP, pois ela define quais são os requisitos de desvio de saúde que devem ser supridos e classifica o sistema de Enfermagem a ser adotado em cada caso. Desta forma, sugere-se a utilização desta nos serviços de nefrologia como suporte teórico para o Processo de Enfermagem.

Salienta-se que este estudo contribui para a prática dos enfermeiros dos serviços de diálise, haja vista que identificou a aplicabilidade da Teoria de Enfermagem de Orem na assistência aos pacientes em diálise peritoneal. Pode orientar estes profissionais sobre a escolha que devem fazer, tornando-se um aporte teórico capaz de fundamentar o Processo de Enfermagem e apoiar a sistematização da assistência, a qual é relevante para reduzir riscos, atenuar sofrimentos, elevar a qualidade do cuidado ofertado e a satisfação de pacientes e famílias. Além disso, a assistência sistematizada contribui para dar visibilidade à atuação resolutiva do enfermeiro no contexto assistencial desta modalidade de serviço de saúde.

Como limitação deste estudo, tem-se a amostra por conveniência que não possibilitou a comparação com outras realidades para afirmações e generalizações. Além da restrição de estudos que versem sobre os requisitos de autocuidado de desvio de saúde e que obstou a discussão da capacidade de autocuidado de pacientes em DP.

Aponta-se a necessidade de novas investigações que abordem a relação entre a capacidade de operacionalização do autocuidado e o atendimento aos requisitos de autocuidado de desvio de saúde sobretudo em outras realidades, para ampliar a discussão sobre esta temática e verificar a aplicabilidade do uso da Teoria de Enfermagem de Orem em outras populações e, assim, generalizar estes achados.

\section{FINANCIAMENTO}

O presente trabalho foi realizado com apoio da Coordenação de Aperfeiçoamento de Pessoal de Nível Superior (CAPES) na modalidade de bolsa de mestrado concedida a Denise Rocha
Raimundo Leone (Código de Financiamento 001). Agradecemos também a Universidade Federal de Juiz de Fora pelo apoio financeiro a esta pesquisa.

\section{CONTRIBUIÇÕES DOS AUTORES}

Desenho do estudo. Denise Rocha Raimundo Leone. Roberta Teixeira Prado. Edna Aparecida Barbosa de Castro.

Coleta ou produção dos dados. Denise Rocha Raimundo Leone.

Análise de dados. Denise Rocha Raimundo Leone. Ana Carolina de Oliveira Jeronymo Neves. Roberta Teixeira Prado.

Edna Aparecida Barbosa de Castro.

Interpretação dos resultados. Denise Rocha Raimundo Leone. Ana Carolina de Oliveira Jeronymo Neves. Roberta Teixeira Prado. Edna Aparecida Barbosa de Castro.

Redação e revisão crítica do manuscrito. Denise Rocha Raimundo Leone. Ana Carolina de Oliveira Jeronymo Neves. Roberta Teixeira Prado. Edna Aparecida Barbosa de Castro.

Aprovação da versão final do artigo. Denise Rocha Raimundo Leone. Ana Carolina de Oliveira Jeronymo Neves. Roberta Teixeira Prado. Edna Aparecida Barbosa de Castro.

Responsabilidade por todos os aspectos do conteúdo e a integridade do artigo publicado. Denise Rocha Raimundo Leone. Ana Carolina de Oliveira Jeronymo Neves. Roberta Teixeira Prado. Edna Aparecida Barbosa de Castro.

\section{EDITOR ASSOCIADO}

\author{
Marta Sauthier
}

\section{REFERÊNCIAS}

1. Woodrow G, Fan SL, Reid C, Denning J, Pyrah AN. Renal Association Clinical Practice Guideline on peritoneal dialysis in adults and children. BMC Nephrol.2017;18(1):333. http://dx.doi.org/10.1186/s12882-0170687-2. PMid:29145808.

2. Neves PDMM, Sesso RCC, Thomé FS, Lugon JR, Nasicmento MM Brazilian Dialysis Census: analysis of data from the 2009-2018 decade. J Bras Nefrol. 2020;42(2):191-200. http://dx.doi.org/10.1590/21758239-jbn-2019-0234. PMid:32459279.

3. Gonzalez-Bedat MC, Rosa-Diez G, Ferreiro A. El Registro Latinoamericano de Diálisis y Trasplante Renal: la importancia del desarrollo de los registros nacionales en latinoamérica. Nefrología Latinoamericana 2017;14(1):12-21. http://dx.doi.org/10.1016/j.nefrol.2016.12.002.

4. Zazzeroni L, Pasquinelli G, Nanni E, Cremonini V, Rubbi I. Comparison of Quality of Life in Patients Undergoing Hemodialysis and Peritoneal Dialysis: a systematic review and meta-analysis. Kidney Blood Press Res. 2017;42(4):717-27. http://dx.doi.org/10.1159/000484115 PMid:29049991.

5. Figueiredo AE, Bernardini J, Bowes E, Hiramatsu M, Price V, Su C et al. A syllabus for teaching peritoneal dialysis to patients and caregivers. Perit Dial Int. 2016;36(6):592-605. http://dx.doi.org/10.3747/pdi.2015.00277. PMid:26917664.

6. Orem DE. Nursing: concepts of practice. 5th ed. Missouri: Mosby; 1995.

7. Campos MXB, Dutra EJO, Silva CJA, Menezes HF, Santos RSC, Silva RAR. Patients undergoing peritoneal dialysis: association between nursing diagnoses and their components. Acta Paul Enferm. 2019;32(6):651-8. http://dx.doi.org/10.1590/1982-0194201900090.

8. Resolução COFEN no 358 de 15 de outubro de 2009 (BR). Dispõe sobre a Sistematização da Assistência de Enfermagem e a implementação 
do Processo de Enfermagem em ambientes, públicos ou privados, em que ocorre o cuidado profissional de Enfermagem, e dá outras providências. O Conselho Federal de Enfermagem (COFEN), no uso de suas atribuições legais que lhe são conferidas pela Lei no 5.905 , de 12 de julho de 1973, e pelo Regimento da Autarquia, aprovado pela Resolucão COFEN no 242, de 31 de agosto de 2000. Diário Oficial da União [periódico na internet], Brasília (DF), 2009 [citado 2020 ago 4]. Disponível em: http://www.cofen.gov.br/resoluo-cofen-3582009_4384. $\mathrm{html}$

9. Bezerra MLR, Faria RPR, Costa de Jesus CA, Dos Reis PED, Pinho DLM, Kamada I. Aplicabilidade da teoria do déficit do autocuidado de ordem no Brasil: uma revisão integrativa. J Manag Prim Health Care. 2019;9. http://dx.doi.org/10.14295/jmphc.v9i0.538.

10. Raimondo ML, Fegadoli D, Méier MJ, Wall ML, Labronici LM, RaimondoFerraz MI. Produção científica brasileira fundamentada na Teoria de Enfermagem de Orem: revisão integrativa. Rev Bras Enferm. 2012 jun;65(3):529-34. http://dx.doi.org/10.1590/S0034-71672012000300020. PMid:23032347.

11. Santos I, Sarat CNF Modalidades de aplicação da teoria do autocuidado de Orem em comunicações científicas de enfermagem brasileira. Rev. enfer. UERJ [Internet]. 2008; [citado 2020 ago 4];6(3):313-8. Disponível em: http:// http://www.facenf.uerj.br/v16n3/v16n3a03.pdf

12. Foster PC, Bannett AM. Dorothea E. Orem. In: George JB. Teorias de enfermagem: os fundamentos à prática profissional. 4a ed. Porto Alegre: Artmed; 2000. p. 83-102.

13. Branco JMA, Lisboa MTL. Tratamento com diálise peritoneal: a prática do autocuidado no contexto familiar. Rev. enferm. Uerj. 2015;23(3):344 9. http://dx.doi.org/10.12957/reuerj.2015.5132.

14. Calderan C, Torres A, Zillmer J, Schwartz E, Silva D. Self-care practices for peoples with chronic renal failure undergoing continuous ambulatory peritoneal dialysis. R. pesq.: cuid. fundam. online. 2013;5(1):3394-3402 http://dx.doi.org/10.9789/2175-5361.2013v5n1p3394.

15. Santos I, Rocha RPF, Berardinelli LMM. Necessidades de orientação de enfermagem para o autocuidado de clientes em terapia de hemodiálise. Rev Bras Enferm. 2011 abr;64(2):335-42. http://dx.doi.org/10.1590/ S0034-71672011000200018. PMid:21755219.

16. Sampaio CF, Guedes MVC. Nursing process as a strategy in the development of competence for self-care. Acta Paul Enferm. 2012;25(2):96103. http://dx.doi.org/10.1590/S0103-21002012000900015.

17. Creswell JW. Projeto de pesquisa: métodos qualitativo, quantitativo e misto. 3a ed. Porto Alegre: Artmed; 2010.

18. Damásio BF, Koller SH. The Appraisal of Self-Care Agency Scale-Revised (ASAS-R): adaptation and construct validity in the Brazilian context. Cad Saude Publica. 2013;29(10):2071-82. http://dx.doi.org/10.1590/0102 311X00165312. PMid:24127101.

19. Stacciarini TSG, Pace AE. Translation, adaptation and validation of a selfcare scale for type 2 diabetes patients using insulin. Acta Paul Enferm. 2014;27(3):221-9. http://dx.doi.org/10.1590/1982-0194201400038.

20. Santos JLG, Cunha KS, Adamy EK, Backes MTS, Leite JL, Sousa FGM. Análise de dados: comparação entre as diferentes perspectivas metodológicas da Teoria Fundamentada nos Dados. Rev Esc Enferm USP. 2018;52:e03303. 2018;52:e03303. http://dx.doi.org/10.1590/ s1980-220x2017021803303. PMid:29668789.

21. Strauss A, Corbin J. Pesquisa qualitativa: técnicas e procedimentos para o desenvolvimento de teoria fundamentada. 2. ed. Porto Alegre: ArtMed; 2008.

22. Resolução no 466, de 12 de dezembro de 2012 (BR). Diretrizes e normas regulamentadoras de pesquisas envolvendo seres humanos. Diário Oficial da União, Brasília (DF), 12 dez 2012.

23. Alcântara FG, Freitas MMS, Furriel AF, Cattafesta M, Salaroli LB Consumo alimentar de pacientes renais crônicos submetidos à diálise peritoneal e fatores associados. Saúde Pesqui. 2020;13(1):63-72. http:// dx.doi.org/10.17765/2176-9206.2020v13n1p63-72.

24. Flores AD, Zillmer JGV, Schwartz E, Lange C, Linck CL, Barcellos CR Support network and social support of people with chronic renal disease under peritoneal dialysis. Rev. pesqui. quali. 2019;7(15):453-72. https:// doi.org/10.33361/RPQ.2019.v.7.n.15.292.

25. Yang F, Luo N, Lau T, Yu ZL, Foo MWY, Griva K. Health-related quality of life in patients treated with continuous ambulatory peritoneal dialysis and automated peritoneal dialysis in Singapore. Pharmacoecon Open. 2018;2:203-8. http://dx.doi.org/10.1007/s41669-017-0046-z.

26. Yu ZL, Lee VY, Kang AW, Chan S, Foo M, Chan CM et al. Rates of intentional and unintentional nonadherence to peritoneal dialysis regimes and associated factors. PLoS One. 2016;11(2):e0149784. http://dx.doi.org/10.1371/journal.pone.0149784. PMid:26919323.

27. Choi S, Ko IS. The Influence of self-care agency and social support on self-care practice among spinal cord injured patients. Korean J Adult Nurs. 2017;29(6):569. http://dx.doi.org/10.7475/kjan.2017.29.6.569.

28. Ademe S, Aga F, Gela D. Hypertension self-care practice and associated factors among patients in public health facilities of Dessie town, Ethiopia BMC Health Serv Res. 2019;19(1):51. http://dx.doi.org/10.1186/s12913019-3880-0. PMid:30665405.

29. Bettoni LC, Ottaviani AC, Orlandi FS. Relationship between self-care and depression and anxiety symptoms in individuals undergoing hemodialysis. Rev. Rene (Online). 2017;18(2):181. http://dx.doi org/10.15253/2175-6783.2017000200006.

30. Armer JM, Henggeler MH, Brooks CW, Zagar EA, Homan S, Stewart BR. The Health Deviation of Post-Breast Cancer Lymphedema: Symptom Assessment and Impact on Self-Care Agency. Self Care Depend Care Nurs. 2008;16(1):14-21. PMID: 22844654.

31. Zillmer JGV, Silva DGV, Mercado-Martinez FJ. My body, my rules! Treatment management in the lives of individuals in peritoneal dialysis. Investigação Qualitativa em Saúde. [Internet]. 2015; [citado 2020 ago 4]; 1:[aprox. 7 telas]. Disponível em: http://proceedings.ciaiq.org/index php/ciaiq2015/article/view/72/69

32. Neitzke DV, Schwartz ES, Zillmer JGV, Lise FL. Perspectives of the person in peritoneal dialysis about the illness process. Rev Par Enferm. [Internet]. 2018; [citado 2020 ago 4];1 (1):[aprox. 9 telas]. Disponível em: http://seer.fafiman.br/index.php/REPEN/article/view/453

33. Lemos PL, Barsaglini R, Paz KMR. Tangible and intangible impacts on the experience of chronic kidney illness. Physis. 2016;26(3):879-99. http://dx.doi.org/10.1590/s0103-73312016000300009.

34. Timm AMB, Beuter M, Girardon-Perlini NMO, Pauletto MR, Santos NO, Backes $C$. Estratégias de (re)organização da família que convive com familiar em diálise peritoneal no domićlio. R. pesq.: cuid. fundam. online. 2017;9(3):696-704. http://dx.doi.org/10.9789/2175-5361.2017. v9i3.696-704.

35. Schmitz J, Bolfe ER, Costa WCS. Itinerário terapêutico e critérios para escolha da diálise peritoneal em pacientes com doença renal crônica. R. Interd. 2018;11(2):17-27. http://dx.doi.org/10.17648\%2F2317-5079. v11n2.1259.

36. Gomes HLM, Monteiro IOP, Pina RMP, Toledo NN, de Almeida GS Enfrentamento, dificuldades e práticas de autocuidado de pacientes com doença renal crônica submetidos à diálise peritoneal. Rev Paul Enferm. 2019;30:1-12. http://dx.doi.org/10.33159/25959484.repen.2019v30a1.

37. Sadala MLA, Bruzos GAS, Pereira ER, Bucuvic EM. Patients' experiences of peritoneal dialysis at home: a phenomenological approach. Rev Lat Am Enfermagem. 2012;20(1):68-75. http://dx.doi.org/10.1590/S010411692012000100010. PMid:22481723.

38. Figueiredo AE, Bernardini J, Bowes E, Hiramatsu M, Price V, Su C et al. A syllabus for teaching peritoneal dialysis to patients and caregivers. Perit Dial Int. 2016;36(6):592-605. http://dx.doi.org/10.3747/pdi.2015.00277. PMid:26917664.

39. Pedroso VSM, Andrade GB, Weykamp JM, Cecagno D, Medeiros AC Siqueira HCH. Ações do enfermeiro na capacitação do usuário e família em diálise peritoneal. R. pesq.: cuid. fundam. online. 2018;10(2):572-6. http://dx.doi.org/10.9789/2175-5361.2018.v10i2.572-576.

40. Naranjo HY, Concepción PJA, Rodríguez LM. La teoría Déficit de autocuidado: Dorothea Elizabeth Orem.Gac. méd. espirit [Internet] 2017; [citado 2020 ago 4];19(3):[aprox. 12 telas]. Disponível em: http:// scielo.sld.cu/pdf/gme/v19n3/GME09317.pdf

\footnotetext{
a Dissertação de mestrado "Diálise Peritoneal Domiciliar: habilidades para a realização do ritual terapêutico" de autoria de Denise Rocha Raimundo Leone, defendida no Programa de Pós-Graduação em Enfermagem da Universidade Federal de Juiz de Fora. No ano de 2016, sob a orientação da Profa. Dra. Edna Aparecida Barbosa de Castro.
} 